\title{
Summary of Bionics Engineering and Its Applications
}

\author{
Zhuojuan Yang ${ }^{1, \text { a }}$ and Zhanyu Xu \\ ${ }^{1}$ Jilin Engineering Normal University, Changchun, China \\ azhuojuan@163.com
}

\section{Keywords: Bionics; Bionic engineering; Application}

\begin{abstract}
Expounds the connotation and research method of Bionics Engineering, introduces the animal bionic information, leaf surface wettability, morphology and function of bionic bionic structure in engineering application, and their research progress and application prospect were analyzed and prospects.Introduction

In the hundreds of millions of years of evolution and co evolution of life in the process of organisms in the micro and macro morphology, structure and function, energy and material transformation, metabolism and utilization system, movement and behavior, heredity, reproduction, development, regulation, assembly process and mechanism, repair, compensation, immune mechanism, information transmission, processing and behavior control ability and the ability to adapt to the environment and so on, through interaction with the environment itself has been optimized, an important source of the biological world of technological innovation.
\end{abstract}

\section{Bionics and Engineering Bionics}

Bionics(Bionics, Biomimetics, Biomimicry) is defined in many ways, and there is not yet a fully unified understanding. Steel, an American military surgeon, first proposed the concept of Bionics in 1960[1-4] . In 1998, the United States published a monograph on the use of bionics bionics Benyus[5] new term (Biomimicry). Bionics is the imitation of biological science, which studies of biological systems, structure, material, function, energy conversion, information control features, and its application in technology system, to improve the existing technology and engineering equipment, the creation process, the new building automation device configuration, such as science, its task is to study the biological system excellent ability, and it mode, and then apply these principle to design and manufacture technology of new equipment. It is a branch of Applied Biology, an interdisciplinary subject in biology, mathematics, and engineering.

Bionics is a specific application of Bionics in engineering technology, it studies various special functions and characteristics of the biological mechanism, explore the ways and methods of its characteristic function imitation used in engineering technology.

The main research methods of bionics and engineering bionics [3,5]: construction model, simulation. The general procedure is as follows: the first part is the study of some excellent functional biological prototype, this biological model; second is the typical characteristic information of the biological prototype provided by mathematical analysis and optimization, we propose a mathematical model with general significance; third is the use of electronics, mathematics, control theory, mechanics, physics, chemistry, material science etc. according to the physical model of mathematical model method, the optimized simulated experiments were carried out on the establishment of engineering technology; finally through improvement and further optimization application in practical engineering field. This process is not a simple imitation, but in the bionic innovation, the final formation of the machine and biological prototype is different, in many ways, even more than the ability of biological models [6-8].

\section{Bionic Information and its Application}

Aquatic Animals. The attachment of marine organisms, such as mussels, to the hull of the ship poses a great threat to the ship and marine facilities. On the one hand, increase the hull weight and hull 
friction resistance, thereby increasing fuel consumption; on the other hand, it will accelerate the corrosion of the hull and shorten the service life of the ship. At present, cuprous oxide coatings in marine and marine facilities in the adhesion of the dominant position, but due to the accumulation of copper in the sea, especially in the sea, resulting in a large number of seaweed deaths, will eventually be disabled. Many countries in the world are committed to the development of a new non polluting anti adhesion coating, the development of bionics may provide a useful way to solve this problem.

Large marine animal such as sharks, dolphins, whales and other skin are not attached to marine organisms, epidermal structure of American and German scientists observe these large marine animal in the electron microscope and found that the existing trench structure in micron their skin, also can secrete a viscous liquid, this structure can prevent the small sea biological or bacteria attached on the surface. The surface structure is simulated by chemical method and some progress has been made. Once the technology breakthrough, anti fouling coatings will become a truly non-toxic antifouling coatings. In addition, there are reports of the use of the above bionic principle to create a swimsuit, sailing ship with reduced resistance to water resistance .

The small feather of the penguin is hollow, and it has a good thermal insulation performance than the solid poultry feathers. Penguins can live in a few degrees below zero in the Antarctic, with its unique feather temperature control capabilities. According to the unique function of penguin feathers, aerospace and energy experts simulating the development of potassium titanate fibers successfully, the fiber has a large number of pores can effectively prevent heat conduction, insulation structure similar to penguin feathers hollow. At present, potassium titanate fiber has become the world's best insulation materials, it is of important scientific and technological achievements of science and technology of bionics .

In the world of marine life, there are many different forms of shells, these shells are not only beautiful appearance, and its structure is also very consistent with the principles of mechanics . For example, some deep sea shells, the appearance of wavy undulating, completely different from the life in the shallow shell on the beach, this is because the sea life in Scallop in Shell hundreds of meters or even thousands of meters under the sea, its shell always bear the great pressure of the water, and the surface geometry of wavy structure can make the shell subjected to compressive strength more in the same thickness, the structure is gradually formed after millions of years of evolution, the force is very scientific and reasonable. In fact, there are countless beautiful natural shapes to imitate, shells, turtle shells, etc., these are the object of imitation and learning.

Water Strider is a common life in ponds, rivers and streams on the surface of insects, it can in the water is free to stop, move and jump. In 2004, Jiang Lei found that the excellent properties of the water strider, not like the previous scholars believe that the effect of surface tension on the leg fat secretion produced, but the use of the leg special micro nano effect to achieve. The water strider leg consists of many setae, the acicular seta diameter from 3 to several hundred nm range, most of the setae length is about 50, and the legs arranged surface tilt 20 degrees direction. The mechanical measurements of the legs show that the maximum support of a leg on the water surface is 15 times the total weight of the body. It is this unique water striderlegs micro nano hierarchical structure to make it in the water move freely, can not sink even in a dangerous situation and rapid flow, this new discovery will help in the near future to design a new type of micro water transport.

Land Animals. The flea jump ability of very high strength, jumping height is 200 times their height, aviation experts have done a lot of research, a British aircraft manufacturing company inspired by the vertical take-off, managed to produce an almost vertical landing harrier aircraft.

House lizard is the most able to crawl. It can easily climb up, upside down cantilever, almost every kind of climbing in the material above, even in the water, vacuum environment and space can walk freely. Found under the microscope, house lizard on the toes about the roots of nanometer fine wool 6 million 500 thousand, each hair diameter of about $200 \sim 500 \mathrm{~nm}$, the diameter of human hair is about $1 / 10$, and the hair is 2 times the length of the diameter of human hair, wool in front of $100 \sim 1000$ similar tree branches of a small fine. The pads of each branch end, can produce very small molecular interactions and surface contact. Although this force is very small, but when all the fine hair on the 
foot of house lizard with the solid surface contact, they produce the total adhesion will exceed the many synthetic adhesive can produce adhesive force. House lizard foot 6 million 500 thousand fine wool all attached to the surface of the object, the weight of the adsorption of $1330 \mathrm{~N}$. By the end of 2003, according to the United States scientists crawl house lizard bionic principle to create a house lizard tape. This study can also be used in aerospace, navigation, underwater exploration, medicine and other important areas.

Soil Animal. The Key Laboratory of ground machine bionics technology of Ministry of education, Jilin University, began to study the theory and technology of some kinds of soil animals and bionics, and obtained a series of research results since the 80 s of last century. For many typical soil animal (such as beetles, voles, earthworms, ants and mole crickets, carabidaes, pangolins) the surface morphology and structure, body fluid, surface potential and surface flexibility is studied experimentally and theoretically analysis system, smooth desorption, drag wear theory and technology of biological non proposed, the research results have been applied in many engineering fields.

Ren Luquan, Chen Bingcong, Tong Jin and other studies in large soil animal indicate that soil animal body exists geometric non smooth features, its characteristic is that certain structural geometry unit randomly or regularly distributed in certain parts of the body, the shape of the structure unit scale shape, convex, concave, ribbed shape and seta, along the longitudinal soil animal body (i.e. movement direction) the undulated surface contour curve. The geometric and non smooth morphology of each part of the same soil animal body surface is different, which is related to the biological evolution process of soil animals to adapt to the environment, especially in different parts of the soil contact. The mechanical properties of geometrically non smooth structures can be characterized as rigid, elastic or flexible. The non smooth structure of the soil animal body surface exists on a variety of scales, and can be divided into macro non smooth, non smooth and non smooth.

The anti sticking function of soil animals is determined by their surface properties, including the geometric shape of the body surface, the surface material composition, the electrical properties of the body surface, body surface flexibility, body surface fluid, etc.. Analysis of the dung beetle, ants and mole crickets, a step surface samples and pangolin scales of the, the outermost layer of soil animal body is a kind of resin wax material, with strong hydrophobicity, the water in the soil animal body surface on the contact angle greater than 90 degrees, the surface soil dynamic hydrophobicity and the body has an important influence on the comprehensive effect of non smooth surface and anti sticking on the surface.

Ren Luquan, Li Jianqiao, Qian Cong of the corrugated and convex non smooth Bionic Bulldozing Plate drag reduction of viscosity, the results show that the biological geometric non smooth surface is the basis of touching soil components of terrain machinery research and design of the bionic surface. The bionic non smooth structure element can produce the normal micro vibration which is beneficial to reduce the tangential adhesion force, and the existence of the non smooth structure makes the adhesive interface distribute discontinuously, which reduces the water film tension. The non smooth surfaces with the same geometric parameters can be used to make the non smooth surfaces with the same geometric parameters, which has a better effect of reducing adhesion and reducing resistance, which can be used as a non smooth structural unit, such as super high molecular weight polyethylene.

Soil animal body surface is flexible. For example, inhabit damp water on the belt body vole coat, many parts of cricket, grasshoppers, crickets, beetles, a step of soil animal are bristle type structure. The soil animal is mainly reflected in the non smooth surface of flexible unit structure, so called biological nonsmooth [28], non smooth structure unit has anti adhesive effect, the unit of force from the flexible soil has the cushioning effect, and through the flexible element is the mutual displacement and distortion of the action the soil has been removed.

The basic idea of flexible bionic imitation is the soil animal body flexible non smooth structure unit, design a series of flexible auxiliary components in mechanical ground touching soil components, such as flexible steel lining cloth and flexible steel chain lining, can make the soil touching parts has significant effect of removal. Wang Yunpeng and Yang Xiaodong of the bionic flexible drag 
reduction system by theoretical analysis and simulation analysis in computer, get the maximum drag reduction rate of $27.8 \%$ flexible drag reduction system; and further to form drag reduction in gas, liquid and solid in drag reduction type and drag reduction mechanism are summarized and analyzed. Ren were sliding resistance test comparison of animal fur flexibility on the surface of different conditions. The results show that under certain conditions, it can improve the elastic deformation energy and reduce the sliding resistance by $10 \% \sim$. Ren through the bionic non smooth surface control rotary structure boundary layer attached to the wall body area to reduce the resistance of rotary body. To have different sizes of convex hull, pits and riblets non smooth slender body and smooth rotating body, low subsonic and supersonic wind tunnel test, according to the analysis of the drag reduction rate, the maximum drag reduction of about $5 \%$.

\section{Surface Wettability and its Application in Plant}

The self cleaning effect of plant leaf surface has aroused people's great interest, for example, lotus leaf, Han Jinlian and some other aquatic plants have the self-cleaning function. By observing the microscopic structure of the leaves of several species of plants, it is believed that the self cleaning feature of the lotus leaf is caused by the presence of the micro structure of the surface and the presence of the surface wax. 1997, two scientists at the University of Bonn, Germany, first discovered and put forward the concept of lotus leaf effect (Lotus Effect), explained the principle of lotus leaf non stick water . In our country, a scholar of China, thinks that there is a nano structure on the surface of the lotus leaf, which is the basic reason of the surface super hydrophobic.

In 2000, the basic principle, the lotus effect based on Klevis and Fraunhof, the German Research Institute developed a self-cleaning surface film material, in Leipzig building materials exhibition of the non stick water anti pollution special plastic film. This film has a lotus leaf micro structure can be used in the family table cloth veneer, it can make furniture surface clean is still exposed to wind and rain, it can be used in solar panels, traffic signs and even roof tile.

Some plants are completely different from other plants, such as lotus leaf, the leaf surface shows good hydrophilic property when it is in contact with water. The main reason for this result is that the surface is not smooth. For example, table velvetleaf with surface morphology is completely different from the lotus leaf, the distribution of many "slender setae", they induce a drop speed exhibit the hydrophilicity of the form. There are a lot of plants (such as taro, etc.) on the surface of the leaves showed good hydrophilic properties, this information on the surface morphology to improve the wettability of the surface provides a new way .

\section{Conclusion and Prospect}

The December 2003 meeting of the Academy of Sciences Chinese Xiangshan conference explicitly put forward the "10 front area in five directions on the biomimetic science and technology system of the foundation and priority development, pointed out the direction for the development of engineering bionics theory research and technology applications.

(1) bionics research platform to build complex biological system, carry out the bionic structure and mechanics, bionic functional devices and control, molecular bionics, micro systems and micro manufacturing technology, bionic brain function and cognition of the five "bionic bionic science and technology basic research direction.

(2) research and development to give priority to the development of biomimetic materials, bionic technology, bionic robot, micro nano bionic technology, gene engineering and tissue engineering, bionic bionic bionic, bionic energy, protein engineering bionics computation and artificial intelligence 10 fronts.

(3) pay attention to the application and commercialization of bionic scientific and technological innovation in the field of the development of the top priority of the high-tech industry in China, so that the overall level of China's bionic science and technology rapidly approaching the developed countries . 
This paper gives a brief overview and analysis of the bionics and application, we can see the development of bionics can provide ways and methods are very important for engineering and technical innovation, believe that combination of bionics and engineering technology will have a broad application prospect.

\section{References}

[1] Ball, P. Lifes lessons in design. Nature, 2001, 409.

[2] Biomimicry--A Review. European Space Agency Work Package Report, 2003: 4-9

[3] Ramachandra. P., Rao. Biomimetics, Sadhana 2003,28( 3\&4): 657-676

[4] Benyus J. M. Biomimicry: Innovation Inspired by Nature. New York: Willianm Morrow and Company, 1998

[5] Anthony T R, Cline H E. Surface Rippling Induced by Surface-Tension Gracients During Laser Surface Melting and Alloying. Journal of Applied Physics, 1997,48(9):3888-3900.

[6] Lancaster J F. Metallurgy of Welding. London: Allen and Unwin Publ., 1990.

[7] M Labudovic, D Hu, R Kovacevic. Three-dimensional finite element modeling of laser sur- face modification. Proc instn mech Engrs, 2000, 214(8): 683-692.

[8] Jiho UH, Jin S. LEE, Yoon H. KIM, et al. Laser engraving of micro-patterns on roll surfaces. ISIJ International,2002,42(11):1266-1272. 